\title{
Design for a Residential Gateway Based on IOT Technology
}

\author{
Shiqiong Guan, Rangding Wang ${ }^{1}$
}

\begin{abstract}
According to the current application of the IOT technology and the development of the remote meter reading, This paper advances a solution scheme of the residential gateway, which is applied to the meter reading area. Maily analyzes the hardware and software design, and the way to realize the communication between the meter reading unit and the residential gateway. In this design, the gate-way communicates with the meter reading unit by the Zigbee RF module, the Zstack-2007 is embedded on the CC2530 chip, to send the received data to the power optical fiber communication network, the gateway takes the s3c2440 as the process unit, which is built upon the UC/OS II operation system, in addition, a fiber optic cat is added to the gateway, in order to convert electrical signals into optical signals. The results show the gateway can work stably and rapidly.
\end{abstract}

Key words: Gateway, Zigbee, UC/OS II, Optical fiber

\section{Introduction}

IOT, the abbreviation of the "internet of things", is a global network infrastruc-ture, linking physical and virtual objects through the exploitation of data capture and communication capabilities. The application of the IOT technology

\footnotetext{
Rangding Wang $(\bowtie)$

College of Information Science and Engineering, Ningbo University, Ningbo, China

wangrangding@nbu.edu.cn
} 
has been used in many fields. Remote meter reading area is one case of the applications, where the residential gateway plays an important role on the kinds of meters. The gateway can be treated as a bridge connecting the internal network and the external network. It is a simple, flexible, intelligent interface unit of the home network and its realization can be described as follows. In case the companies need to send some instructions to control or read meters, such as water meter, gas meter, and so on, the residential gateway will convert the received instructions from the external network to the data in the zigbee network, and more, the data reading from the meters will be sent to the gateway at regular time.

Presently, the modes of the remote meter reading can be classified as wired and wireless. The wired mode contains 485, M-Bus, CAN, ADSL and power line carrier. Among them, the power line carrier does not exist the wiring problems and has low cost advantage, but the reliability of the signal is inevitably affected by the interference of the strong electric field during the transmission[1]. The wireless remote meter reading mode contains GRPS and ad-hoc network. Due to the high cost of GPRS, it's not easy to get promoted[2]. Therefore, ZigBee technology as the ad-hoc network is adopted in this design. The system structure of the proposed residential gateway is shown in Fig.1.

The design of the proposed residential gateway is based on the CC2530 and S3C2440. It is primarily focusing on the remote meter reading area, including wa-ter, gas and heat meters. It has the potential to reduce the cost and improve the work efficiency.

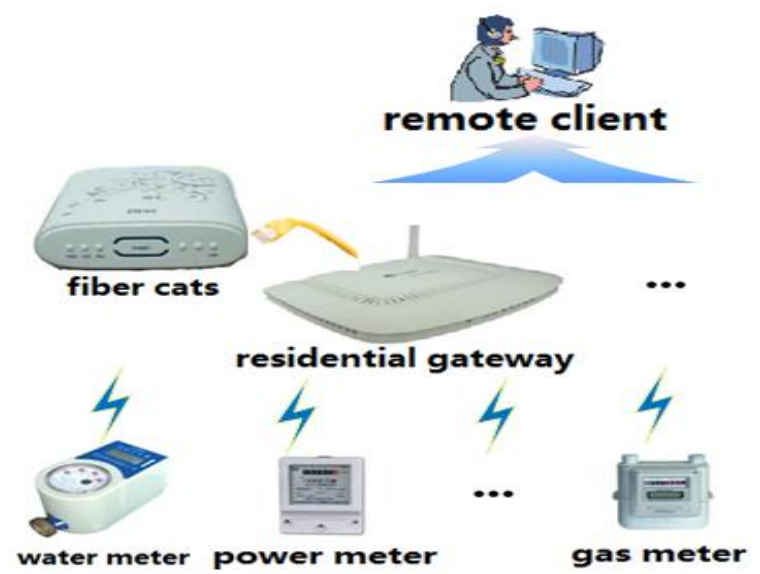

Fig 1. Residential gateway system structure 


\section{System Hardware Design}

\subsection{Overall Design of the System}

The design of the residential gateway contains the following several modules, such as RF modules, MCU, Memory module, Ethernet access module, Power supply module, Clock module, LED and buttons module. The hardware block diagram is shown in Fig.2.

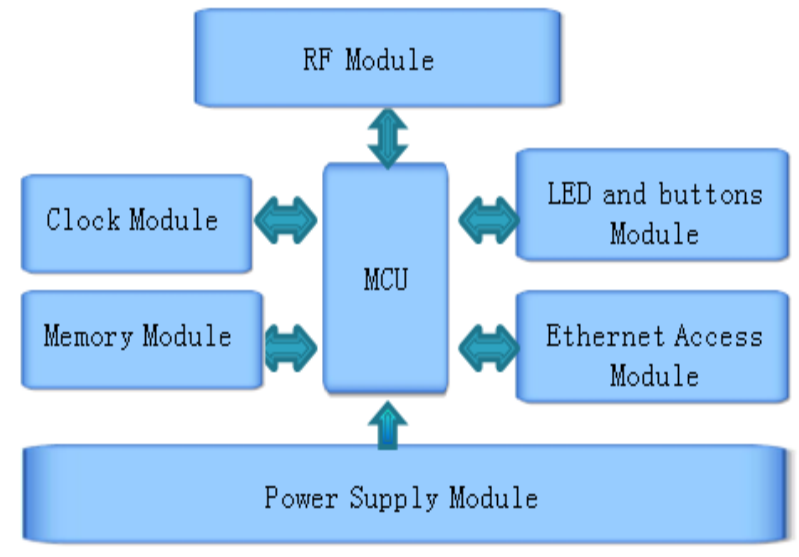

Fig 2. Hardware block diagram

In the clock module, a $12 \mathrm{Mhz}$ passive crystal is selected as the clock source. A reset circuit is used in this design to make sure the chip can be reset normally. In addition, a $256 \mathrm{M}$ off-chip flash and 64M SDRAM storage are added to the cir-cuit, The flash is used to store Bootloader、Linux and other system files, while the SDRAM is used to store running programs and data. In the power supply cir-cuit, the modules wildly used by the engineers contain adapter powered supply and battery powered supply. In this design, battery powered supply module is adopted, meanwhile a bidirectional level translator chip (74LVC4245) is selected to provide the required voltage supply for the circuit. To make the gateway work in a more efficient and low cost energy way. Besides, a battery management unit is included in this module. In the power supply unit of the ZigBee, it is designed to operate for long time by entering sleep mode for much of its life and only waking up periodically to send the meter reading instructions, or respond to the interrupt 
from the ending devices. Two LEDs and a joystick button are used in the LED and button circuit. The colors of the LEDs are green and yellow, which are used to show the work states of the gateway. The joystick button can be used as a five buttons module, since it owns five directions of the operation, left, right, up, down and push.Ethernet access realizes the function to make the gateway get access to the internet and it plays an important part in this design. A whole Ethernet access circuit includes an Ethernet controller chip, a network filter and a RJ45 interface. The RTL8019AS is selected as the Ethernet controller chip. It works in full-duplex module to meet the requirement of the transmission rate in the design. The $20 \mathrm{~F} 001 \mathrm{~N}$ is a twisted pair/driver receiver chip and chosen as the network filter, Since there are two transmitting transformers on the $20 \mathrm{~F} 001 \mathrm{~N}$, it plays a role in connecting the Ethernet controller to the RJ45 interface[3].

Since most families surf the internet through the optical fiber network in place of the Ethernet. And the optical fiber network is more safe and reliable[4], so in order to connect the gateway with the optical fiber network, the fiber cats is used to link with the RJ45 interface and optical fiber interface, it helps convert electricity signals into light signals.

\subsection{RF Module}

The RF module contains RF chip and RF power amplifier. The CC2530 is selected as the RF chip and CC2591 as the RF power amplifier chip.

The CC2530 produced by TI is a kind of wireless single chip based on the 2nd generation compatible with the ZigBee/IEEE802.15.4 standard. It has a strong anti-interference and a wide working voltage range. Additionally, the CC2530 owns extensive hardware support, including packet snooping 、 data buffering 、 burst transmission、data encryption、data authentication、 channel cleanup assessment 、 linking quality indication、 packet timestamp, etc, the CC2530 to-gether with the S3C2440 form a double-CPU, which makes the gateway work on a more effective and reliable condition than the popular single-CPU system.

In the RF power amplifier unit, the CC2591 is adopted, which has low power consumption and can achieve longer distance transmission at a lower power supply in combination with the CC2530 chip. The circuit connection diagram is 
shown in Fig.3.

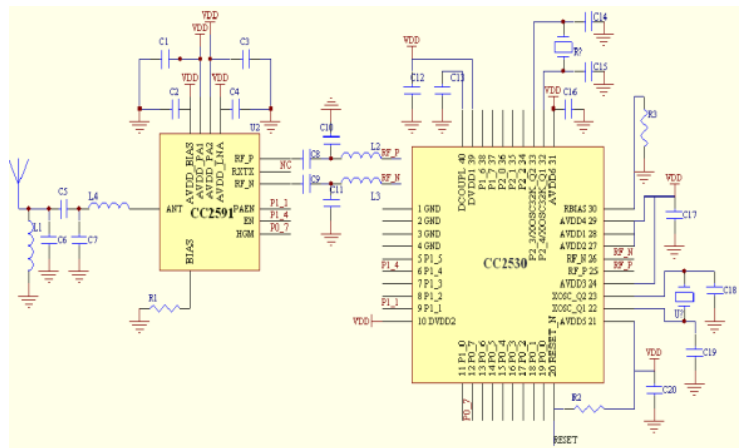

Fig 3. RF circuit connection diagram

The CC2530 RF unit, the S3C2440 and the Ethernet controller chip are con-nected by the serial ports. The function of the S3C2440 in this design is protocol translation, date sending and receiving.

The communication between the RF module and the residential gateway realized with a SPI interface. It includes FIFOP, CCA, SFD, SI, SO, SCLK, CSn. The CC2530 uses SFD, FIFO, FIFOP, CCA four pins to query the status of data sending and receiving, and uses $\mathrm{CSN}, \mathrm{SO}, \mathrm{SI}, \mathrm{SCK}$ to transfer data, instructions with the $\mathrm{S} 3 \mathrm{C} 2440$.

\section{System Software Design}

\subsection{RF Tansformat}

The residential gateway communicates with the meter reading modules by the star network configuration, each meter is one node of the network. it is a high efficient communication mode. It has two ways To send or receive the data in the gateway, one is to take the initiative to upload, the ending module transimites the data at regular time. the other way is roll polling, the ending module makes corresponding operations once the gateway sends the reading or writing instructions to the ending devices. The initiative uploading way is adopted in this paper. 
MAC frame types include beacon frame, data frame, command frame and response frame.the zigbee frame types adopted here is command frame, data frame. The command frame is to transmit instructions to read data from the meters, or control the ending devices. The data read from the meters will send to the gateway by data frame type. The frame types are shown in table 1 .

Table 1. Data frame format

\begin{tabular}{|c|c|c|c|c|c|c|c|c|}
\hline & $\begin{array}{c}\text { Frame } \\
\text { Heade } \\
\mathrm{r}\end{array}$ & $\begin{array}{c}\text { Lengt } \\
\mathrm{h}\end{array}$ & $\begin{array}{c}\text { Short } \\
\text { Addres } \\
\mathrm{s}\end{array}$ & $\begin{array}{c}\text { Data } \\
1\end{array}$ & $\begin{array}{c}\text { Data } \\
2\end{array}$ & $\cdots$ & $\begin{array}{c}\text { Data } \\
\mathrm{M}\end{array}$ & $\begin{array}{c}\mathrm{CR} \\
\mathrm{C}\end{array}$ \\
\hline $\begin{array}{c}\text { Numbe } \\
\mathrm{r} \text { of } \\
\text { bytes }\end{array}$ & 1 & 1 & 2 & $1+\mathrm{N}$ & $1+\mathrm{N}$ & $\begin{array}{c}1+ \\
\mathrm{N}\end{array}$ & $1+\mathrm{N}$ & 0 \\
\hline Content & $\mathrm{FB}$ & $\mathrm{xx}$ & HHLL & $\mathrm{xx}$ & $\mathrm{xx}$ & $\mathrm{xx}$ & $\mathrm{xx}$ & 0 \\
\hline
\end{tabular}

Since the gateway adopts the SPI communication interface to communicate with the RF module. So the CRC will not be used. The Frame Header defaults to be FB, it occupies small storage space of 1 byte. The length of the data will be added from Data1 to DataM.

A 64-bit IEEE address is put on the Data1, the format is $81[\mathrm{xx} \mathrm{xx} \mathrm{xx} \mathrm{xx}$ $\mathrm{xx} x \mathrm{xxx} x \mathrm{x}$ ], the value of the RSSI is put on the Data2, format is $23[\mathrm{xx}]$.

The data read from meters, or the instructions send to the end devices, are put on the left frame unit. Take the water meter reading as an example, suppose the collected data of the frame is put on Data3, since four bits of the type definition uses 32-bit analog symbols, the number is 6 , the number of the four bits is also 6 , it indicates that the data is user-defined, the latter data is the value of the reading, format is 66[XXXX.XX].

The meters can be controlled by PC through the remote network, the frame type of the command can be supposed to be put on Data4, take the instruction of controlling a meter for example. The head of the bit must be set 0 , and the back 8 -bit is set 1 , so the frame format of the command is 01 [xx]. 


\subsection{RF Communication Unit}

To meet the requirements of the practical application, the Star Network structure is selected in this design. The data sending and receiving on the $\mathrm{CC} 2530$ are run-ning in the TXFIFO and RXFIFO registers. The data is written into the TXFIFO before sending. Once the data is received, the interrupt occurs. Then the data is read out from the RXFIFO in the interrupt service routine. The data is sent out by three modes, (1) slotted CSMA/CA. (2) non-slotted CSMA/CA. (3) not CSMA/CA. Because the slotted CSMA/CA mode requires the end device and the gateway to be synchronized[5], the non-slotted CSMA/CA mode is chosen in this design. The result of some researchers' experiment under this mode shows there's no packet loss within the communication radius when the polling time is greater than $15 \mathrm{~ms}[7]$. The flow chart of the CC2530 is shown in Fig.4.

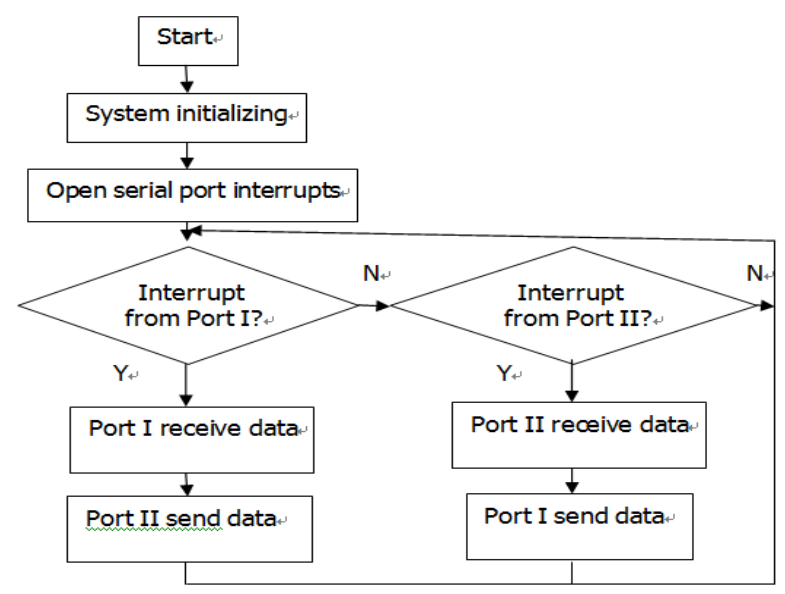

Fig 4. Data sending and receiving flow chart on the CC2530

During the data sending and receiving, a sleep mode is required since the system is not at work in most cases. The z-stack 2007 is transplanted into the CC2530 and provides two sleep modes: TIMER sleep and DEEP sleep. The TIMER sleep is set to make the CC2530 work in a lower energy cost. Once the interrupt occurs, the CPU of the CC2530 is woken up. Then the system enters the work mode, and calls the interrupt subroutine. After the interrupt is handled or the data is sent out, the system goes back to the sleep mode. The operating current of the CC2530 in sleep mode stays in uA level[6].which makes the gateway almost no energy cost in the cc2530 in the 
most of the time.

\section{Design of the Embedded System}

The tasks of the software design of the embedded system contain uC/OS II and Lwip transplant、Data sending and receiving、Protocol conversion.

To begin with the design of the embedded system, the boot code of the S3C2440 should be finished firstly. its process goes as follows: reset, then set the application Entry Point and interrupt vector table, initialize the memory, clock cir-cuit, peripheral port, application memory space, execution space, loading space, data space, zero initialized space, at last boot the $\mathrm{uC} / \mathrm{OS}$ II operating system to complete enabling the chip. For those unused interrupts, they are required to point to the function with a return instruction when setting the interrupt vector table. Meanwhile the stack pointer should be defined firstly to avoid the system confu-sion brought by the error interrupt. In addition, since the S3C2440 does not have memory system, so adopt to initialize the register instead of to initialize the mem-ory system.

Compared with Linux, uC/OS II is the most widely used embedded operating system, and it is more simply to be transplanted, and more effectively to work. Meanwhile, the gateway designed based on the $\mathrm{uC} / \mathrm{OS}$ II is more stable and reli-able[8]. The operating system is transplanted into the S3C2440 to realize protocol conversion and some other tasks scheduling.

The TCP/IP protocol is transplanted into the designed system. The traditional TCP / IP protocol is too complex because its realization brings higher requirements on the memory and computational speed, so an embedded TCP / IP protocol stack is chosen here. There are many kinds of open source embedded TCP/IP protocols, such as Lwip, uC/IP, ILIP, etc. Among these embedded protocols, the Lwip proto-col stack has a simple user interface and supports for sockets direct protocol, data receiving and sending by using the API functions provided by the protocol stack[9]. Its protocol layer is divided clearly, what's more, the Lwip is primarily designed based on the full consideration of the future transplantation. The sections related with the operation system, hardware and compiler are placed under the content of the /src/arch file[10]. The transplantation 
of the Lwip is to edit the files under the content. it's easy for engineers to start the design based on the protocol. The transplant of the protocol stack contains several parts as follows:

(1) Write header files related with the CPU.

(2) Write functions related with the operation system.

(3) Write interface functions related with the hardware.

In addition, the semi-open source protocol Z-stack 2007 produced by the TI is transplanted into the CC2530 chip.

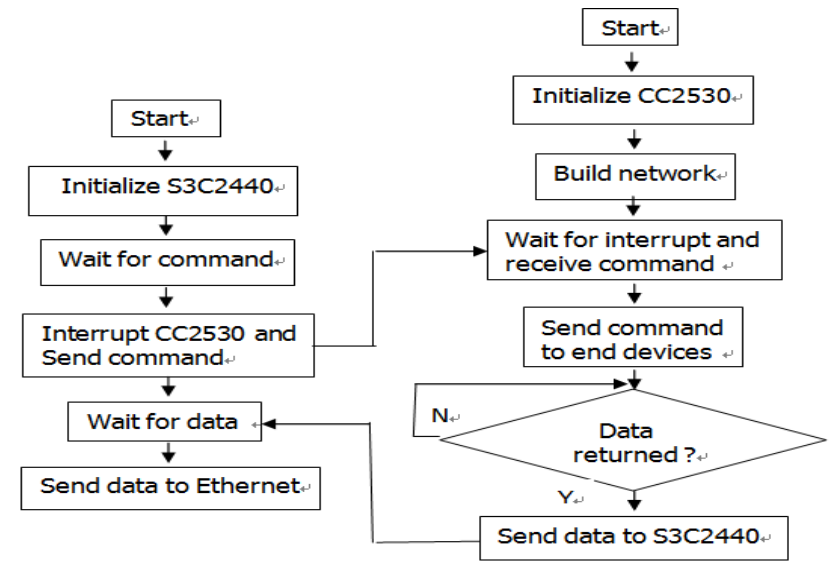

Fig 4. Data sending and receiving flow chart on the $\mathrm{S} 3 \mathrm{C} 2440$

The protocol conversion is realized on the $\mathrm{S} 3 \mathrm{C} 2440$ chip. its realization process can be described as follows.

(1)The ZigBee module packets the received data, and then sends the packaged data to the $\mathrm{S} 3 \mathrm{C} 2440$ via a serial port.

(2) On the application layer of the gateway, the data packaged will be sent up to the ARP layer by layer, then the

MAC address is snatched from the ZigBee network by the ARP

(3)The MAC address is converted into the IP address of the TCP/ IP network, and then the packaged data is sent to the TCP handler function of the TCP/IP network to deal with dividedly,

(4)The package data is sent down to the MAC address on the network inter-face layer,

Thus the protocol conversion is finished from the ZigBee network to the TCP / IP network, and the principle of converting the TCP / IP network to the ZigBee 
network is similar to the process above.

\section{Conclusion}

In this paper, the design of a residential gateway based on the IOT technology is proposed, which is mainly focusing on the meter reading area. The hardware and software schemes are provided by the consideration on the feasibility.

In the test process, we firstly do the packet loss rate, set the value of transmitted power as $4 \mathrm{dbm}$. And set the packet transmission rate as $100 \mathrm{p} / \mathrm{s}$, On the condition of sending 1000packets, the result of the test is shown in table 2. Case1: under the condition in the open space; Case2:under the condition through one wall; Case3: under the condition through two walls.

Table 2. test of zigbee packet loss rate

\begin{tabular}{|l|l|l|l|l|l|l|}
\hline $\begin{array}{c}\text { Transmission } \\
\text { distance } \\
(\mathrm{m})\end{array}$ & $5 \mathrm{~m}$ & $10 \mathrm{~m}$ & $15 \mathrm{~m}$ & $20 \mathrm{~m}$ & $25 \mathrm{~m}$ & $30 \mathrm{~m}$ \\
\hline Case1 & 1000 & 1000 & 1000 & 1000 & 1000 & 1000 \\
\hline Case2 & 1000 & 993 & 939 & 825 & 701 & 544 \\
\hline Case3 & 997 & 922 & 781 & 422 & 118 & 95 \\
\hline
\end{tabular}

The conclusion shown in table 2 is the successfull transmission rate can reach no less than $80 \%$, wheather pass through one wall or through two walls. It can be realized under normally use in most families.

On power consumption, under the state of hibernation mode $1,2,3$, sending and receiving data. The measured current in reality is shown in table 3. From the table, the value of the current is only $0.51 \mathrm{uA}$, when the mode is entering the hibernation mode 3 . What's more, the current of the data sending and receiving is also in the range of the withstand. 
Table 3 test of power consumption under the $\mathrm{rf} \mathrm{rx} / \mathrm{tx}$ mode

\begin{tabular}{|l|c|l|l|l|l|}
\hline & $\begin{array}{l}\text { Hibernation } \\
\text { mode 1 }\end{array}$ & $\begin{array}{l}\text { Hibernation } \\
\text { mode 2 }\end{array}$ & $\begin{array}{l}\text { Hibernation } \\
\text { mode 3 }\end{array}$ & $\begin{array}{l}\text { Receiving } \\
\text { mode }\end{array}$ & $\begin{array}{l}\text { Sending } \\
\text { mode }\end{array}$ \\
\hline $\begin{array}{l}\text { Current } \\
\text { in } \\
\text { theory } \\
(\mathrm{mA})\end{array}$ & 0.2 & 0.001 & 0.00045 & 10 & 17 \\
\hline $\begin{array}{l}\text { Current } \\
\text { In fact } \\
(\mathrm{mA})\end{array}$ & 0.226 & 0.00103 & 0.00051 & 16.424 & 28.17 \\
\hline
\end{tabular}

The analysis and result shows the design can realize the data transmission and protocol translation. In the future work, some related experiments will be done to strengthen the performance of the designed gateway, make sure it can work on a more stable, reliable and low power comsumption condition in the meter reading area.

\section{Acknowledgement}

This work is supported by the Zhejiang science \& technology preferred projects of China (2010C11025), Zhejiang province education department key project of China (ZD2009012).

\section{References}

1,Xiaolan Wang, Zhongbiao Zhao, Weiping Liu. The Design of Remote Reading Meters Based on CAN/RS485. Microcomputer Information. 2006(62):113-115.

2,Zihong Zhang, Hongyan Zhou. The Design of Wireless Meter Reading System Based on Zig-Bee Technology. Telecommunications for Electric Power System. 2012(2):60-63.

3,Zhixue Wang, Hailong Wang, Junhua Song. The Application in Ethe $\neg r\urcorner$ net Communication Based on Network Card Chip RTL8019AS[J]. New Technology Application.2009(4):15-17. 
4,Shenzhen guodian communication technology company. Introduction of the smart use of power in a housing estate based on PFTTH[J]. Digital Community \& Smart Home. 2010(6):56-60.

5,Shuli Hao,Xianguo Tuo, Dashun Xi. Design of Landslide Monitoring System Based on CC2530[J].Electronic Design Engineering .2011.19(15):40-42.

6,Ren Huang, Hui Hao, Junhua Ren. Analysis and Research of Unslotted CSMA/CA for IEEE802.15.4. Computer Engineering and Application. 2009.45(7): 108-110.

7,Zhifang Li, Hongsheng Zhong. Pointtopoint Communication Based on CC2530 and IEEE 802.15.4[J]. Microcontrollers \& Embedded Systems. 2011,11(7):43-45.

8,Liuqiang Zhao, Yongjun Lin, Liangyu Ma, Xinya Chen. Date Acquis $\neg$ i $\neg$ tion System Based on uC/OSII Embedded System[J].Microcomputer Information.2010(5):65-66.

9,Yajun Wang. A lightweight TCP/IP Protocol Stack Lwip Applied in the Embedded System[J]. Computer Era.2008(5):15-16.

10,Longteng $\mathrm{Hu}, \mathrm{Yu}$ Tian. Design and Implementation of System for Embedded Ethernet Net-work Based on LWIP[J]. Digital Technology \& Application. 2010(7): 44-44. 